\title{
Diagnóstico da comercialização de madeira serrada no estado de Mato Grosso
}

\author{
Leonardo Antônio de Moraes ZAQUE ${ }^{1 *}$, Rafael Rodolfo de MELO ${ }^{1,2}$, \\ Diego Martins STANGERLIN ${ }^{1,3}$, Laercio SERENINE JUNIOR ${ }^{1}$
}

\author{
${ }^{1}$ Programa de Pós-Graduação em Ciências Florestais e Ambientais, Universidade Federal de Mato Grosso, \\ Cuiabá, MT, Brasil. \\ ${ }^{2}$ Departamento de Ciências Agronômicas e Florestais, Universidade Federal Rural do Semi-Árido, Mossoró, RN, Brasil. \\ ${ }^{3}$ Instituto de Ciências Agrárias e Ambientais, Universidade Federal de Mato Grosso, Sinop, MT, Brasil. \\ *E-mail: leonardo_zaque@hotmail.com
}

Recebido em agosto/2018; Aceito em fevereiro/2019.

\begin{abstract}
RESUMO: O presente estudo teve como objetivo avaliar o comércio madeireiro no estado de Mato Grosso, analisando as principais espécies madeireiras comercializadas e os seus principais produtos gerados. Os dados foram coletados a partir dos relatórios de Beneficiamento e Comércio de Produtos da Madeira por Espécie Florestal e disponibilizados pela Secretaria de Estado do Meio Ambiente de Mato Grosso (SEMA-MT). O diagnóstico foi realizado no período entre 2007 e 2014, onde foram analisados o volume total, os valores comercializados das dez espécies mais comercializadas e dos seus produtos gerados. As dez espécies mais comercializadas (Erisma uncinatum Warm., Goupia glabra Aubl, Qualea albiflora Warm., Qualea paraensis Ducke, Mezilaurus itauba, Qualea sp., Hymenolobium sp., Apuleia sp., Trattinnickia sp. e Vochysia sp.) obtiveram um valor de 5,6 bilhões e um volume aproximado de 10,42 milhões de metros cúbicos no período, que corresponde a $48,60 \%$ do volume total comercializado. Os produtos analisados corresponderam a quase $36 \%$ de todos os produtos comercializáveis pelas principais espécies em volume, e em valor corresponderam a cerca de $70 \%$ do total.
\end{abstract}

Palavras-chave: indústria madeireira; madeira tropical; comércio de madeiras.

\section{Diagnosis of the commercialization of sawn timber in the state of Mato Grosso}

\begin{abstract}
The present study aimed to evaluate the timber trade in the state of Mato Grosso, analyzing the main timber species traded and its main products generated. The data were collected from the reports of Beneficiation and Trade of Madeira Products by Forest Species and made available by the State Secretariat of Environment of Mato Grosso (SEMA-MT). The diagnosis was made between 2007 and 2014, where the total volume, the commercialized values of the ten most traded species and their products were analyzed. The ten most commercialized species (Erisma uncinatum Warm., Goupia glabra Aubl, Qualea albiflora Warm., Qualea paraensis Ducke, Mezilaurus itauba, Qualea sp., Hymenolobium sp., Apuleia sp., Trattinnickia sp. e Vochysia sp.) obtained a value 5.8 billion and an approximate volume of 10.42 million cubic meters in the period, which corresponds to $48.60 \%$ of the total volume traded. The products analyzed corresponded to almost $36 \%$ of all products traded by the main species in volume, and in value corresponded to about $70 \%$ of the total.
\end{abstract}

Keywords: timber industry; tropical wood; timber trade.

\section{INTRODUÇÃO}

O Brasil é o principal produtor e consumidor de madeira serrada tropical, e no ano de 2013, o país produziu cerca de 16 milhões em metros cúbicos de madeira serrada tropical e consumiu aproximadamente 15,8 milhões (Organización Internacional de las Maderas Tropicales - OIMT, 2015). A produção madeireira do estado de Mato Grosso possui inquestionável importância para o Brasil, pois além de ser um grande produtor de madeira serrada, o estado ainda produz lâminas, chapas de compensado, entre outros produtos (ARO; BATALHA, 2013).

A madeira serrada pode ser definida como peças produzidas a partir do desdobro de toras por meio de serras, representando um tipo de transformação primária da madeira. Dependendo do formato e das dimensões estas peças podem ter várias denominações, tais como: vigas, tábuas, pranchas, pontaletes, sarrafos, ripas e caibros (Associação Brasileira da Indústria de Madeira Processada Mecanicamente - ABIMCI, 2009). Geralmente, a madeira serrada, sem beneficiamento, utiliza poucos recursos tecnológicos, agrega menor valor e se destina principalmente ao mercado nacional (Serviço Florestal Brasileiro e Instituto do Homem e Meio Ambiente da Amazônia - SFB/IMAZON, 2010).

Ribeiro et al. (2016), estudando o comércio de madeiras no estado de Mato Grosso, verificaram que as dez espécies mais comercializadas em ordem decrescente no período de 2004 a 2010 em termos de volume foram: Qualea sp. (cambará), Goupia glabra Aubl. (cupiúba), Erisma uncinatum Warm. (cedrinho), Mezilaurus itauba Meissn. (itaúba), Hymenolobium sp. (angelim), Apuleia sp. (garapeira), Manilkara sp. (maçaranduba), Cordia goeldiana Huber (freijó), Dipteryx sp. (cumbarú) e Trattinnickia sp. (amescla), respondendo por $88 \%$ do total comercializado, as quais geraram uma receita de aproximadamente $\mathrm{R} \$ 4,81$ bilhões.

Segundo Ribeiro (2013), nesse período, o estado de São Paulo constituiu-se como o maior consumidor de madeira serrada oriunda de Mato Grosso, consumindo mais de 2,70 milhões de $\mathrm{m}^{3}$. Seguindo, destacaram-se os estados de Santa 
Catarina (1,69 milhões de $\left.\mathrm{m}^{3}\right)$, Paraná $\left(900,46 \mathrm{mil} \mathrm{m}^{3}\right)$, Rio Grande do Sul (722,24 mil m³), Rio de Janeiro (390,16 $\left.\mathrm{mil} \mathrm{m}^{3}\right)$, Mato Grosso do Sul $\left(361,04 \mathrm{mil} \mathrm{m}^{3}\right)$ e Minas Gerais $(279,69$ mil $\mathrm{m}^{3}$ ). Esses sete estados juntos consumiram $95,40 \%$ do volume total de madeira comercializada pelo estado de Mato Grosso.

Desse modo, o presente estudo teve como objetivo avaliar o comércio madeireiro no estado de Mato Grosso, entre os anos de 2007 e 2014, analisando as principais espécies madeireiras comercializadas e os seus principais produtos gerados.

\section{MATERIAL E MÉTODOS}

\section{1. Área de estudo}

O diagnóstico foi realizado com dados do estado de Mato Grosso e avaliou as principais espécies comercializadas entre o período de 2007 a 2014. O estado de Mato Grosso pertence à região Centro-Oeste do Brasil e possui área territorial aproximada de 903,37 mil km², com cerca de 3,03 milhões de habitantes e um PIB (Produto Interno Bruto) per capita de R\$ 23,2 mil. A economia está alicerçada nas atividades agropecuárias, industriais, comerciais e madeireiras. Essa última que impulsionou de forma direta a economia de dezenas de municípios mato-grossenses localizados na Amazônia (Secretaria de Estado de Planejamento e Coordenação Geral SEPLAN, 2013).

\subsection{Coleta de dados}

Os dados foram coletados nos relatórios sobre Beneficiamento e Comércio de Produtos da Madeira por Espécie Florestal e disponibilizados online pela Secretaria de Estado do Meio Ambiente de Mato Grosso (SEMA-MT, 2016), através da emissão de Guias Florestais.

O Decreto 8.189, de 10 de outubro de 2006 do estado de Mato Grosso foi criado para disciplinar a utilização, o preenchimento e a emissão da Guia Florestal (GF) para o transporte de produtos e, ou subprodutos de origem florestal as quais são subdivididas em cinco categorias, GF-1, GF-2, GF3, GF3i e GF-4 (MATO GROSSO, 2006). A descrição de cada uma destas categorias é apresentada a seguir:

$\checkmark$ A GF-1 é usada para o transporte de produtos de origem florestal (tora) efetuado desde a origem até a indústria.

$\checkmark$ A GF-2 é utilizada para o transporte de produtos e, ou subprodutos de origem florestal oriundos de: Plano de Manejo Florestal Sustentável (PMFS), Plano de Exploração Florestal (PEF), Desmate Autorizado em Licenças de Instalação (DALI), Desmate Autorizado em Pequenas Propriedades (DAPP), Exploração Florestal em Pequenas Propriedades (EFPP), Produto Florestal de Limpeza de Pastagens (PFLP), Produto Florestal de Declaração de Estoque (PFDE), Reflorestamento com Espécies Nativas (RCEN), Reflorestamento com Espécies Exóticas (RCEE), Erradicação ou Poda de Cultura ou Espécie Frutífera (EPCF) e Corte ou Poda de Árvores Urbanas (CPAU), como: carvão, lenha; toretes, escoramentos, postes não imunizados, palanques roliços, mourões ou moirões, lascas, palmitos de origem nativa, com exceção do babaçu Orbignya oleifera Bur, oriundo de pastagem e cultura agrícola e mudas.

$\checkmark$ A GF3 é usada para o transporte dos seguintes produtos e, ou subprodutos de origem florestal como: madeira serrada bruta ou semiacabada, produtos semiacabados, produtos beneficiados, produtos industrializados, toras nas hipóteses de revenda para qualquer pessoa jurídica cadastrada no Cadastro de Consumidores de Matéria-Prima de Origem Florestal (CCSEMA), resíduos de produtos florestais oriundos de indústrias, os produtos e, ou subprodutos florestais da GF-2, na segunda operação e carvão originário de resíduos industriais.

$\checkmark \quad$ A GF3i: é utilizada para transporte interestaduais.

$\checkmark$ A GF-4 é usada nos casos em que não couber a emissão das Guias Florestais Modelos 1, 2 e 3, e ainda, para aqueles que não tenham obrigatoriedade de serem cadastrados no CC-SEMA. É exigida também nos seguintes casos: transferência de produtos florestais entre estabelecimentos produtores pertencentes ao mesmo proprietário ou entre proprietários diversos, mas que tenham a mesma participação societária, doações e aquisições eventuais de produtos e, ou subprodutos de origem florestal oriundos de propriedades menores ou iguais a 150 ha (cento e cinquenta hectares).

\subsection{Análise dos dados}

Para análise dos dados apenas as GF3 e GF3i foram usadas, que são utilizadas para o transporte dos produtos e, ou subprodutos de origem florestal.

Para as dez espécies mais comercializadas foram coletados os volumes em metros cúbicos e os valores em reais dos seus produtos, durante o período de 2007 a 2014. Os produtos (Tabela 1) também foram analisados separadamente: caibro, pranchão, prancha, ripa, sarrafo, tábua, viga e vigota, conforme estabelecido pelas normas NBR 7203 (ABNT, 1982) e NBR 14807 (ABNT, 2002).

Tabela 1 - Nomenclaturas e dimensões de peça de madeira segundo as normas da ABNT.

Table 1 - Nomenclatures and dimensions of wood according to ABNT standards.

\begin{tabular}{ccc}
\hline Nome da & \multicolumn{2}{c}{ Dimensões - Espessura/Largura $(\mathrm{cm})$} \\
\cline { 2 - 3 } peça serrada & NBR 7203/1982 & NBR 14807/2002 \\
\hline Caibro & $4,0-8,0 / 5,0-8,0$ & $4,0-8,0 / 5,0-8,0$ \\
Prancha & $4,0-7,0 />20,0$ & $3,9-7,0 />16,1$ \\
Pranchão & $>7,0 />20,0$ & $7,1-16,1 />16,1$ \\
Ripa & $<2,0 /<10,0$ & $1,0-2,0 / 2,0-5,0$ \\
Sarrafo & $2,0-4,0 / 2,0-10,0$ & $2,1-3,9 / 2,0-9,9$ \\
Tábua & $1,0-4,0 />10,0$ & $1,0-3,7 />10,0$ \\
Viga & $>4,0 / 11,0-20,0$ & $4,0-8,0 / 8,1-16,0$ \\
Vigota & $4,0-8,0 / 8,0-11,0$ & Não especificado \\
\hline
\end{tabular}

Fonte: NBR 7203 (1982); NBR 14807 (2002).

$\mathrm{Na}$ análise foram descartadas as madeiras exóticas e a classificação "Diversos", já que não foi informado no banco de dados as espécies utilizadas, nem a especificação dos tipos de produtos transportados. Também foram descartados as toras e produtos que são comercializados em metro estéreo e em quilos.

Os dados foram analisados a partir dos somatórios dos valores e volumes totais comercializados anualmente. Foram identificadas, ao longo do período de estudo, as principais espécies comercializadas e seus produtos gerados, bem como, os seus valores médios $\left(\mathrm{R} \$ / \mathrm{m}^{3}\right)$ e porcentagem.

\section{RESULTADOS}

3.1. Diagnóstico da comercialização

Entre 2007 e 2014 as espécies mais comercializadas em volume foram: Erisma uncinatum Warm., Goupia glabra, 
Qualea albiflora Warm., Qualea paraensis Ducke, Mezilaurus itauba, Qualea sp., Hymenolobium sp., Apuleia sp., Trattinnickia sp. e Vochysia sp., com 10,42 milhões de metros cúbicos no período avaliado (Tabela 2).

$\mathrm{O}$ valor total obtido para as dez principais espécies no período de 2007 a 2014 foi de aproximadamente 5,6 bilhões de reais, uma média de 700 milhões de R\$/ano. Entre 2004 e 2010, para o mesmo estado, Ribeiro et al. (2016) encontraram um valor de 4,05 bilhões de reais, média de 577 milhões de $\mathrm{R} \$$ /ano.

As dez principais espécies do presente estudo corresponderam a $48,60 \%$ do volume total em metros cúbicos (Figura 1). Já Ribeiro et al. (2016), entre 2004 e 2010, encontraram um volume em metros cúbicos que corresponde a $78,14 \%$ para as mesmas dez espécies mais comercializadas do presente trabalho.

Essa redução foi expressiva, demonstrando que houve busca de uma exploração mais diversificada de espécies madeireiras, sendo esse resultado uma sinalização de mudança positiva. Segundo o Instituto de Pesquisas Tecnológicas do Estado de São Paulo - IPT (2013), a crescente utilização de espécies alternativas, fruto de informações disponibilizadas por meio do estudo das propriedades de novas espécies com potencial madeireiro, continuem para que que espécies antes desconhecidas figurem como novas alternativas para o mercado.

Tabela 2. Valores totais, volumes totais e valor médio para as principais espécies madeireiras comercializadas no estado de Mato Grosso no período de 2007 a 2014

Table 2. Total values, total volumes and average value for the main timber species traded in the state of Mato Grosso from 2007 to 2014.

\begin{tabular}{lcccc}
\hline \multicolumn{1}{c}{ Espécies } & Nome comercial & Valor (em bilhões de R\$) & Volume (em milhões de $\left.\mathrm{m}^{3}\right)$ & Valor médio $\left(\mathrm{R} \$ / \mathrm{m}^{3}\right)$ \\
\hline Erisma uncinatum & Cedrinho & 1,52 & 2,46 & 616,16 \\
Qualea sp & Cambará & 0,89 & 2,18 & 409,57 \\
Goupia glabra & Cupiúba & 0,84 & 1,57 & 535,77 \\
Mezilaurus itauba & Itaúba & 0,61 & 0,76 & 798,2 \\
Hymenolobium sp & Angelim & 0,51 & 0,89 & 574,23 \\
Apuleia sp & Garapeira & 0,46 & 0,78 & 581,94 \\
Trattinnickia sp & Amescla & 0,43 & 0,96 & 453,27 \\
Qualea albiflora & Cambará & 0,12 & 0,26 & 464,81 \\
Qualea paraensis & Cambará & 0,11 & 0,25 & 452,65 \\
Vochysia sp & Cambará-rosa & 0,12 & 0,32 & 366,34 \\
\hline \multicolumn{1}{c}{ Total } & & 5,6 & 10,42 & 537,78 \\
\hline \multicolumn{1}{c}{} & & &
\end{tabular}

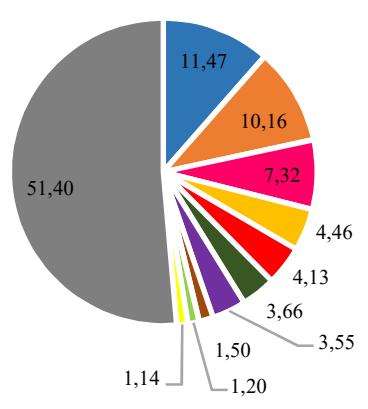

$$
\begin{aligned}
& \text { - Erisma uncinatum } \\
& \text { - Qualea sp } \\
& \text { - Goupia glabra } \\
& \text { - Trattinickia sp } \\
& \text { - Hymenolobium sp } \\
& \text { - Apuleia sp } \\
& \text { - Mezilaurus itauba } \\
& \text { - Vochysia sp } \\
& \text { - Qualea albiflora } \\
& \text {-Qualea paraensis } \\
& \text { - Outras espécies }
\end{aligned}
$$

Figura 1. Porcentagem do volume das principais espécies comercializadas no período de 2007 a 2014 no Mato Grosso.

Figure 1. Percentage of the volume of the main species marketed in the period 2007 to 2014 in Mato Grosso.

Na Tabela 3 são apresentados os dados anuais do volume total comercializado e o volume parcial (dez espécies mais comercializadas), seguida pela representação percentual destas, para o período de 2007 a 2014. Observa-se que no ano de 2007 ocorreu o maior volume comercializado (3,44 milhões de $\mathrm{m}^{3}$ ); já nos anos posteriores ocorreu queda na comercialização de madeiras, provavelmente devido à crise imobiliária americana. Ainda no ano de 2007, as principais espécies comercializadas corresponderam a $32,34 \%$ deste total, a menor proporção na série temporal avaliada.

$\mathrm{Na}$ Figura 2 são apresentados os volumes anuais das espécies mais comercializadas em cada ano. A madeira da espécie Erisma uncinatum foi a mais comercializada durante todo o período analisado, porém teve queda nas vendas no ano de 2014 de 19\% em relação ao ano de 2007. Já as espécies Hymenolobium sp., Mezilaurus itauba, Trattinnickia sp., Apuleia sp. e Vochysia sp. obtiveram quedas de 11\%, 44\%, $58 \%, 42 \%$ e $97 \%$, respectivamente, no ano de 2014 em relação a 2007 .
Tabela 3. Volume total comercializado e volume das dez espécies mais representativas e sua proporção em relação ao volume total, durante o período de 2007 a 2014 no de Mato Grosso.

Table 3. Total market volume and volume of the ten more representative species and its proportion in relation to total volume, during the period 2007 to 2014 in Mato Grosso.

\begin{tabular}{cccc}
\hline \multirow{2}{*}{ Ano } & $\begin{array}{c}\text { Volume total } \\
\text { comercializado } \\
\left.\text { (milhões de } \text { m }^{3}\right)\end{array}$ & \multicolumn{2}{c}{$\begin{array}{c}\text { Total das dez espécies mais } \\
\text { comercializadas }\end{array}$} \\
\cline { 3 - 4 } & 3,44 & 1,11 & Milhões m \\
\hline 2007 & 2,76 & 1,33 & 32,34 \\
2008 & 2,43 & 1,37 & 48,29 \\
2009 & 2,58 & 1,51 & 56,30 \\
2010 & 2,54 & 1,43 & 58,54 \\
2011 & 2,54 & 1,34 & 56,28 \\
2012 & 2,70 & 1,28 & 52,89 \\
2013 & 2,46 & 1,05 & 47,46 \\
2014 & 21,45 & 10,42 & 42,54 \\
\hline Total & & &
\end{tabular}

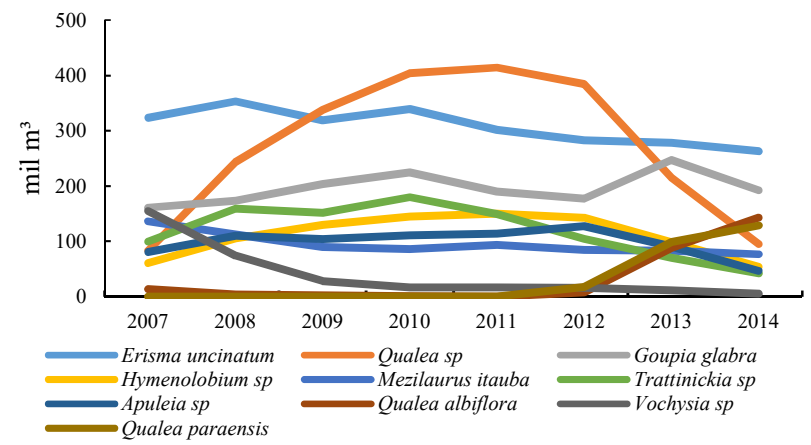

Figura 2. Volumes anuais das principais espécies comercializadas no Mato Grosso no período de 2007 a 2014.

Figure 2. Anual volumes of major species marketed in Mato Grosso in the period 2007 to 2014 . 


\subsection{Produtos madeireiros gerados}

De acordo com Aro e Batalha (2013) a produção de madeira serrada compreende uma série de peças de dimensões padronizadas em relação a sua a largura, espessura e comprimento, gerando os seguintes produtos: tábuas, vigas, pranchas, pranchões, vigotas, sarrafos, ripas e caibros.

$\mathrm{Na}$ Figura 3 são apresentados os volumes $\left(\mathrm{m}^{3}\right)$ produtos obtidos das principais espécies florestais comercializadas no Mato Grosso no período de 2007 a 2014. Na Tabela 4 são apresentados os valores, volumes e valor $/ \mathrm{m}^{3}$ dos produtos madeireiros das principais espécies comercializadas; e na Figura 4 são apresentados os valores em reais desses produtos por ano.

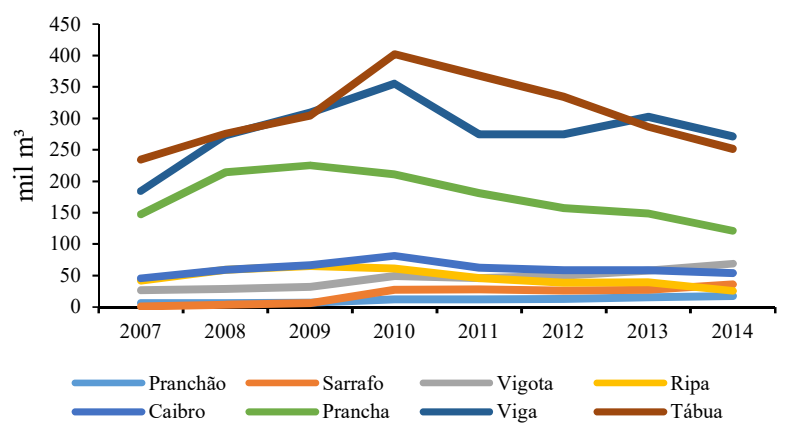

Figura 3. Volume dos produtos madeireiros comercializados pelas principais espécies no Mato Grosso entre 2007 e 2014.

Figure 3. Volume of wood products marketed by major species in Mato Grosso between 2007 and 2014.

Tabela 4. Valores, volumes e valor $/ \mathrm{m}^{3}$ dos produtos madeireiros das principais espécies comercializadas no estado de Mato Grosso no período de 2007 a 2014.

Table 4. Values, volumes and value $/ \mathrm{m}^{3}$ of the wood products of the main species marketed in the state of Mato Grosso in the period from 2007 to 2014 .

\begin{tabular}{|c|c|c|c|c|c|}
\hline \multirow[b]{2}{*}{ Produtos } & \multicolumn{2}{|c|}{ Valor } & \multicolumn{2}{|c|}{ Volume } & \multirow[b]{2}{*}{$\begin{array}{c}\text { Valor/ } \\
\mathrm{m}^{3}\end{array}$} \\
\hline & $\begin{array}{c}\text { Bilhões } \\
\text { R\$ }\end{array}$ & $\%$ & $\begin{array}{c}\text { Milhões } \\
\mathrm{m}^{3}\end{array}$ & $\%$ & \\
\hline Pranchão & 0,056 & 0,99 & 0,089 & 0,41 & 626,80 \\
\hline Sarrafo & 0,073 & 1,30 & 0,155 & 0,72 & 468,62 \\
\hline Ripa & 0,139 & 2,48 & 0,377 & 1,76 & 368,80 \\
\hline Vigota & 0,190 & 3,40 & 0,359 & 1,67 & 530,28 \\
\hline Caibro & 0,254 & 4,54 & 0,486 & 2,27 & 523,07 \\
\hline Prancha & 0,732 & 13,07 & 1,407 & 6,56 & 520,55 \\
\hline Viga & 1,139 & 20,32 & 2,246 & 10,47 & 507,13 \\
\hline Tábua & 1,330 & 23,74 & 2,457 & 11,46 & 541,41 \\
\hline Outros produtos & 1,691 & 30,17 & 13,871 & 64,68 & 121,91 \\
\hline Total & 5,604 & & 21,45 & & 261,31 \\
\hline
\end{tabular}

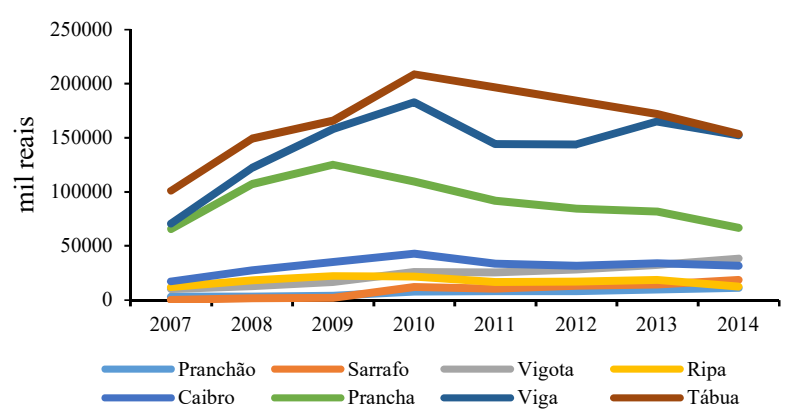

Figura 4. Valores em reais dos produtos madeireiros comercializados pelas principais espécies no Mato Grosso entre 2007 e 2014.

Figure 4. Real value of wood products marketed by major species in Mato Grosso between 2007 and 2014.

\section{DISCUSSÃO}

Os maiores valores e volumes totais comercializados foram obtidos pela Erisma uncinatum, Qualea sp. e Goupia glabra (Tabela 2). Essas espécies também foram as mais comercializadas em volumes totais no período de 2004 a 2010 no estado de Mato Grosso (Ribeiro et al., 2016). Provavelmente foram as mais comercializadas por apresentarem maior qualidade, disponibilidade e aceitação de mercado.

Apesar da Mezilaurus itauba ser a quarta mais comercializada, foi a que apresentou maior valor médio por metro cúbico. Já a Qualea sp. foi a segunda mais comercializada, entretanto o valor médio por metro cúbico situou-se entre os menores, junto com Qualea albiflora, Qualea paraensis, Trattinnickia sp e Vochysia sp.

Reis (2017) explica que apesar das vantagens econômicas obtidas pela comercialização de madeiras, o uso excessivo de um pequeno grupo de espécies pode promover a sua extinção. A grande procura por madeiras estabelecidas no mercado pode resultar na superexploração, causando uma queda brusca na abundância de espécies das áreas manejadas.

De acordo Melo et al. (2015) as principais espécies exploradas em Planos de Manejo Florestal Sustentável (PMFS) e Planos de Exploração Florestal (PEF) na cidade de Sinop, importante polo madeireiro da região Norte do Mato Grosso, no período de 2006 a 2013, foram Qualea paraensis, Erisma uncinatum, Trattinnickia rhoifolia Willd., Apuleia leiocarpa (Vogel) J.F Macbr., Mezilaurus itauba, Micropholis sp. e Goupia glabra. Espécies semelhantes às deste estudo, exceto pela presença de Micropholis sp. Esses autores afirmaram que a grande parte do volume explorado é representado por poucas espécies, o que sugere que os recursos florestais precisam ser melhor utilizados.

Conforme o censo florestal realizado por Oliveira (2014) em uma região de Floresta Ombrófila Densa de Terra-Firme primária localizada no município de Santa Carmem - MT, as espécies que mais se destacaram por ocorrer em um número relevante foram Qualea albiflora, Erisma uncinatum, Trattinnickia burserifolia, Goupia glabra e Mezilaurus itauba. Estas mesmas espécies estão entre as mais comercializadas no presente estudo, ou seja, são espécies que apresentam maiores diversidades de usos.

Em 2010, houve maior comercialização das principais espécies, porém nos anos posteriores ocorreu menos participação das principais espécies, e em 2014, obtiveram o menor volume de comercialização, indicando que essas espécies estão sendo substituídas por outras. Isso pode ser explicado pela incorporação de espécies alternativas empregadas na construção civil, em contraposição aos impactos ambientais causados pelo uso intensivo e constante de determinadas espécies, que se traduz em um importante passo do setor (Zenid, 2009).

Em trabalhos realizados na região de Florianópolis - SC, no período de 2010 a 2011, o estado de Mato Grosso foi o que forneceu o maior volume de madeira serrada de origem tropical (Santos et al., 2014). Segundo estes mesmos autores, as espécies mais comercializadas foram: Andira sp., Dinizia sp., Vatairea sp., Pithecellobium sp., Hymenolobium sp., Vochysia sp., Qualea sp., Erisma sp., Erisma uncinatum, Apuleia sp. e Mezilaurus itauba. Isso confirma que as espécies Erisma uncinatum, Qualea sp., Hymenolobium sp., Apuleia sp., e Vochysia sp. estão entre as mais comercializadas do estado. 
As espécies que apresentaram aumento na comercialização no ano de 2014 em relação a 2007 foram Goupia glabra, Qualea sp., Qualea albiflora e Qualea paraensis com 19\%, $15 \%, 968 \%$ e $63156 \%$, respectivamente, havendo destaque para as duas últimas, que apresentaram incremento significativo nos últimos anos, a partir de 2012. Essa evolução pode ser explicada pela diversidade de espécies manejadas, uma característica relevante do manejo de florestas nativas na Amazônia Legal (Zerbini, 2014).

Apesar da Qualea sp. ter apresentado aumento na comercialização em volume em 2014 em relação a 2007, obteve queda acentuada no ano de 2013, provavelmente isso ocorreu pelo fato das espécies Qualea albiflora e Qualea paraensis passarem a ser identificadas. Antes, estas duas espécies compunham unicamente o gênero Qualea.

Verifica-se que os menores volumes de produtos comercializados foram nos anos de 2007 e 2014, ocorrendo um aumento na comercialização após 2008. Segundo a United States Departament of Agriculture - USDA (2010), os produtos madeireiros estão cada vez mais sendo exigidos por arquitetos, designers de produtos e proprietários para utilizarem na construção civil por serem originados de uma fonte sustentável.

Os anos em que se observa maior produção dos produtos de madeira serrada das principais espécies foram entre 2009, 2010 e 2011, com mais de um milhão de metros cúbicos para cada ano. E segundo Santos et al. (2013), a produção de madeira serrada no estado de Mato Grosso nesse período correspondeu a $31 \%, 35 \%, 24 \%$, respectivamente, da produção da Amazônia Legal, ficando atrás apenas do estado do Pará nos anos entre 2009 e 2011, que obteve 44\%, 34\% e $39 \%$. Conforme o Instituto Brasileiro de Geografia e Estatística - IBGE (2012), em 2010, a economia brasileira foi influenciada por um cenário internacional favorável, sobretudo pelo fortalecimento da demanda interna, com elevação da renda das famílias e maior oferta de crédito, que elevaram o resultado do Produto Interno Bruto (7,5\%), sendo a maior taxa desde 1986 (7,5\%). Consequentemente, a atividade da construção no PIB cresceu 11,6\%, atingindo 5,7\% de participação.

A tábua foi o produto mais comercializado durante o período, porém sua venda oscilou muito, atingindo seu ápice em 2010 e queda na nos anos seguintes. De acordo Carvalho (2016), as tábuas, que tem forma retangular, geram peças de tamanho menor, por possuir aplicação tanto na construção civil como no setor moveleiro. Essa versatilidade pode explicar a maior procura por esse produto.

Dos produtos estudados, a tábua, viga e prancha foram os principais produtos comercializados no período, correspondendo juntos a $28 \%$ do volume total dos produtos das principais espécies, o que equivale a mais de 6,0 milhões em metros cúbicos. Porém, esses produtos foram os que mais oscilaram durante o período. De 2009 a 2014 a comercialização da prancha caiu todos os anos, já a tábua teve queda a partir de 2010. Provavelmente essa queda ocorreu pela substituição da madeira nativa por outros materiais como alumínio, plásticos e MDF (Medium Density Fiberboard) que começaram a ser utilizados demasiadamente em esquadrias, formas de construção civil e móveis (VERÍSSIMO; PEREIRA, 2014).

O caibro, pranchão, ripa, vigota e sarrafo foram os produtos menos comercializados, apresentando juntos $6,83 \%$ do volume total das principais espécies, equivalendo a 1,46 milhões em metros cúbicos. Contudo, em relação a 2007, para o sarrafo, pranchão e vigota houve um crescimento na comercialização desses produtos. Os outros produtos que são representados por compensados, madeira beneficiada entre outros produtos, apresentaram maior volume comercializado durante o período.

Conforme a Tabela 4, os produtos que apresentaram os maiores volumes foram a tábua, a viga e a prancha, que representaram juntas $57 \%$ do valor das principais espécies comercializadas no estado de Mato Grosso entre 2007 e 2014. Já o pranchão, sarrafo, viga, vigota e caibro obtiveram 13\% do valor das principais espécies comercializadas.

O pranchão foi o produto menos comercializado, porém foi o produto com maior valor por metro cúbico, sendo o produto mais valorizado durante o período, provavelmente devido a sua maior dimensão, que possibilita obter peças menores. Já para os outros produtos observou-se tendência contrária, ou seja, foram os mais comercializados, entretanto o preço por metro cúbico foi menor.

\section{CONCLUSÕES}

As dez espécies mais comercializadas representaram metade do volume total comercializado, o que reflete pequeno número de espécies, frente ao potencial madeireiro do estado.

As espécies mais comercializadas foram Erisma uncinatum, Qualea sp. e Goupia glabra. A madeira de Mezilaurus itauba foi a que apresentou o maior valor em metro cúbico, e a Vochysia sp., o menor.

Os produtos mais comercializados corresponderam a quase $36 \%$ de todos os produtos comercializáveis pelas principais espécies em volume, e em valor corresponderam a cerca de $70 \%$ do total.

Os produtos mais comercializados em volume e valor foram viga, tábua e prancha; e os menos comercializados foram pranchão, sarrafo e ripa.

\section{REFERÊNCIAS}

ABIMCI_ASSOCIAÇÃO BRASILEIRA DA INDÚSTRIA DE MADEIRA PROCESSADA MECANICAMENTE. Estudo setorial 2009 Ano Base 2008 - Indústria de Madeira Processada Mecanicamente. Curitiba: ABIMCI, 2009. 43 p.

ASSOCIAÇÃO BRASILEIRA DE NORMAS TÉCNICAS ABNT. NBR 7203/1982: Madeira serrada e beneficiada. Rio de Janeiro: ABNT, 1982. 2 p.

ASSOCIAÇÃO BRASILEIRA DE NORMAS TÉCNICAS ABNT. NBR 14807/2002: Peças de madeira serrada dimensões. Rio de Janeiro: ABNT, 2002. 2 p.

ARO, E. R.; BATALHA, M. O. Competitividade da madeira serrada do Estado de Mato Grosso - Brasil. Gestão e Regionalidade, São Caetano do Sul, v. 29, n. 87, p.81-94, set/dez. 2013.

CARVALHO, D. E. Melhoria no desdobro em uma serraria de eucalipto para madeira destinada a construção. 2016. 83f. Dissertação (Mestrado em Engenharia Florestal) - Universidade Federal do Paraná, Curitiba, 2016

IBGE INSTITUTO BRASILEIRO DE GEOGRAFIA E ESTATÍSTICA. Pesquisa Anual da Indústria na Construção, Rio de Janeiro, v. 20, p. 1-96, 2010.

IPT INSTITUTO DE PESQUISAS TECNOLÓGICAS DO ESTADO DE SÃO PAULO. Catálogo de madeiras 
brasileiras para a construção civil. São Paulo: IPT, 2013. $103 \mathrm{p}$.

MATO GROSSO GOVERNO DO ESTADO DE MATO GROSSO. Decreto 8.189 DE 10 DE OUTUBRO DE 2006. Disciplina a Guia Florestal (GF) para o transporte de produtos e, ou subprodutos de origem florestal do Estado de Mato Grosso, e dá outras providências. Diário Oficial do Estado do Mato Grosso, 10 de outubro de 2016.

MELO, R. R.; PASTORE, K. C.; MASCARENHAS, A. R. P.; ACOSTA, F. C.; PEDROSA, T. D.; SERENINI JUNIOR, L. Vouchers for Releasing Forestry Credit (CLCF) for Sinop, Mato Grosso, Brazil. Nativa, Sinop, v. 3, n. 1, p. 36-43, 2015. DOI: http://dx.doi.org/10.14583/23187670.v03n01a06

OLIVEIRA, M. F. Critérios para o manejo sustentável de duas espécies madeireiras das florestas tropicais do Mato Grosso. 2014. 127f. Dissertação (Mestrado em Ciências Florestais) - Universidade Federal do Paraná, Curitiba, 2014.

OIMT ORGANIZACIÓN INTERNACIONAL DE LAS MADERAS TROPICALES. Reseña bienal y evaluación de la situación mundial de las maderas 2013-2014. Yokohoma: OIMT, 2015. 223 p.

REIS, P. C. M. R. Análise estrutural e propriedades tecnológicas da madeira de espécies da Amazônia. 2017. 94f. Tese (Doutorado em Ciências Florestais) Universidade Federal de Viçosa, Viçosa, 2017.

RIBEIRO, E. S. Comercialização de madeira serrada de florestas naturais em Mato Grosso: um diagnóstico do setor de base florestal. 2013. 116f. Dissertação (Mestrado em Ciências Florestais e Ambientais - Universidade Federal de Mato Grosso, Cuiabá, 2013.

RIBEIRO, E. S.; SOUZA, R. A. T. M.; PAULA, M. H.; MESQUITA, R. R. S.; MOREIRA, E. L.; FAZION, H. Espécies florestais comercializadas pelo estado de Mato Grosso. Biodiversidade, Rondonópolis, v. 15, n. 2, p.2-20, maio/ago. 2016.
SANTOS, A. S.; SILVA, F. A. P. R. C.; SIMONETTI, R. A.; ROBERT, R. C. G.; FANTINI, A. C. Panorama do comércio de madeira serrada na Microrregião Geográfica de Florianópolis - SC. Floresta e Ambiente, Seropédica, v. 21 , n. 1, p.19-29, jan/mar. 2014. DOI: http://dx.doi.org/10.4322/floram.2013.035

SANTOS, D.; PEREIRA, D.; VERÍSSIMO, A. O estado da Amazônia: uso da terra. Belém: IMAZON, 2013. 70 p.

SEMA-MT_SECRETARIA DE ESTADO DO MEIO AMBIENTE DE MATO GROSSO. Beneficiamento e Comércio de Produtos da Madeira por Espécie Florestal. 2016. Disponível em: $<$ http://www.sema.mt.gov.br/portalsisflora/index.php/rela torios>. Acesso em: 10 ago. 2016.

SEPLAN SECRETARIA DE ESTADO DE PLANEJAMENTO E COORDENAÇÃO GERAL. Mato Grosso em números - Um diagnóstico da realidade de Mato Grosso. Cuiabá: SEPLAN, 2013. 181 p.

SFB SERVIÇO FLORESTAL BRASILEIRO; IMAZON INSTITUTO DO HOMEM E MEIO AMBIENTA DA AMAZÔNIA. A atividade madeireira na Amazônia brasileira: produção, receita e mercados. Belém: SFB/IMAZON, 2010. 20 p.

USDA_UNITED STATES DEPARTAMENT OF AGRICULTURE. Wood handbook - Wood as an engineering material. Madison: USDA, 2010. 509 p.

VERÍSSIMO, A.; PEREIRA, D. Produção na Amazônia Florestal: características, desafios e oportunidades. Parcerias Estratégicas, Brasília, v. 19, n. 38, p. 13-44, jan/jun. 2014.

ZERBINI, F. Cenário da Madeira FSC no Brasil 2012 2013. São Paulo: FSC Brasil, 2014. 81 p.

ZENID, G. J. Madeira uso sustentável na construção civil. 2. Ed. São Paulo: IPT, 2009. 99 p. (Publicação IPT, 3010). 